www.jmscr.igmpublication.org

Index Copernicus Value: 79.54

ISSN (e)-2347-176x ISSN (p) 2455-0450

crossrefDOI: https://dx.doi.org/10.18535/jmscr/v7i1.152

\title{
Multiple Epidermoid Cysts in the Palatine Tonsil Causing Referred Otalgia: A Case Report
}

\author{
Authors \\ Dr Amrita Suzanne Mathew ${ }^{1 *}$, Dr B.V Chandre Gowda ${ }^{2}$, Dr Aishwarya Sridhar ${ }^{3}$ \\ ${ }^{1}$ Assistant Professor, ${ }^{2}$ Professor and HOD \& Vice Principal, ${ }^{3}$ Post Graduate \\ Department of ENT, MVJ Medical College and Research Hospital, Hoskote, Bangalore-562114, \\ Karnataka, India \\ *Corresponding Author \\ Dr. Amrita Suzanne Mathew
}

Assistant Professor, Department of ENT, MVJ Medical College and Research Hospital, Hoskote,

Bangalore-562114, Karnataka, India

Email:amritasmathew@gmail.com

\section{Abstract}

Tonsillar retention cysts are relatively common in clinical practice. Rarer varieties such as epidermoid cysts are usually solitary and present with complaints such as "lump in the throat" or difficulty swallowing. We therefore report a case diagnosed with multiple epidermoid cysts who presented with the unusual feature of referred otalgia.

Keywords: Epidermoid cyst;Palatine tonsil;Referred otalgia; Glossopharyngeal nerve.

\section{Introduction}

Among the different types of tonsillar cysts, retention cysts are the commonest, while epidermoid cysts, lymphoepithelial cysts and hydatid cysts are rarer. ${ }^{1}$ Epidermoid cysts (EC) are benign lesions histologically characterized by cystic spaces lined by squamous epithelium. They can occur in any part of the body with approximately $1.6-6.9 \%$ of cases arising in head and neck area, of which only $13.33 \%$ were EC, while $58.88 \%$ were dermoid cysts. The incidence of intraoral EC is only $0.01 \%$; mainly found in the floor of the mouth (sublingual, submental or submandibular areas) or labial, lingual, buccal mucosa. EC in the pharynx is a rare presentation and particularly tonsillar cysts are a rare occurrence. $^{2}$

Referred/secondary otalgia is a challenging symptom, with the burden on the physician to identify the source. It occurs in up to $50 \%$ of adults consulting a general physician for otalgia, and because of the nature of the ear's sensory innervations, a wide variety of disorders can produce referred otalgia. Referred otalgia transmitted by the glossopharyngeal nerve may be secondary to lesions and/or inflammatory processes of the nasopharynx, palatine tonsil, soft palate, or posterior one-third of the tongue. ${ }^{3}$

Review of literature revealed cases with a single tonsillar EC with dysphagia/odynophagia being the primary symptom. ${ }^{1,2,4} \mathrm{We}$ therefore report an 


\section{JMSCR Vol||07||Issue||01||Page 861-864||January}

unusual case of multiple intratonsillar epidermoid cysts presenting with referred otalgia.

\section{Case Report}

A 32 year old female presented to the Department of Otorhinolaryngology with pain in the left ear for the past 1 year, which was dull aching type, continuous and aggravated on swallowing. No other associated symptoms/pertinent complaints were present. Otological examination was normal. With a provisional diagnosis of referred otalgia, further examination for the source of pain was carried out. On oral examination a yellowish, well circumscribed, soft swelling was identified over the medial aspect of the left tonsil, measuring about $1.5 \times 1.0 \mathrm{~cm}$, along with multiple soft swellings, each about $0.5 \times 0.5 \mathrm{~cm}$ in the upper pole. (Fig.1) Examination of the neck was normal. Bilateral tonsillectomy was performed. On gross examination, the specimen showed multiple swellings as seen on clinical examination. Histopathological examination revealed tonsillar tissue within which were cavities lined by stratified squamous epithelium, containing lamellated keratin flakes, macrophages and lymphocytes, characteristic of intratonsillar ECs. (Fig.2) Post operatively, her earache was relieved and follow-ups were normal.

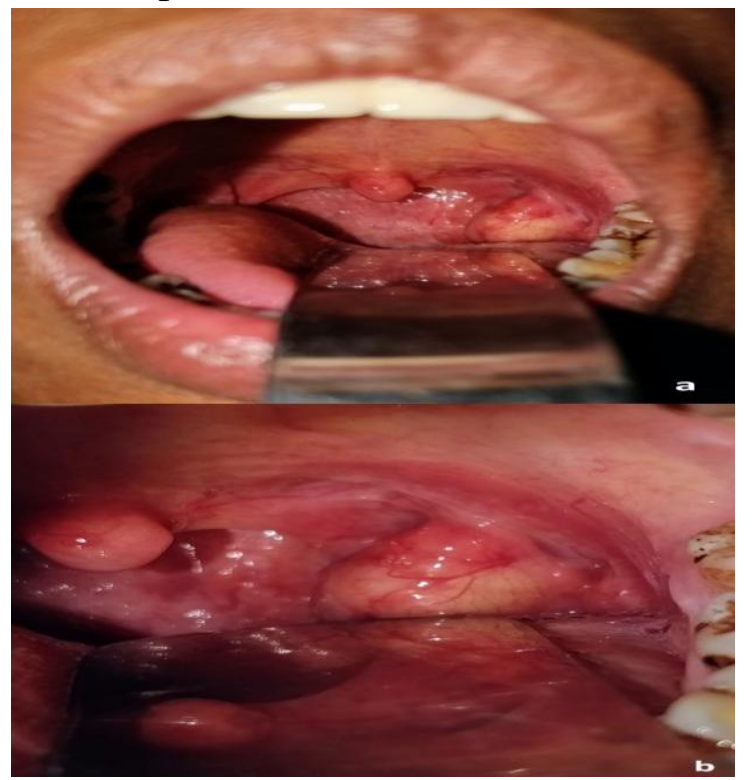

Fig.1 Clinical images a Yellowish swelling over medial aspect of left tonsil. b Closer inspection of the left tonsil revealed multiple swellings

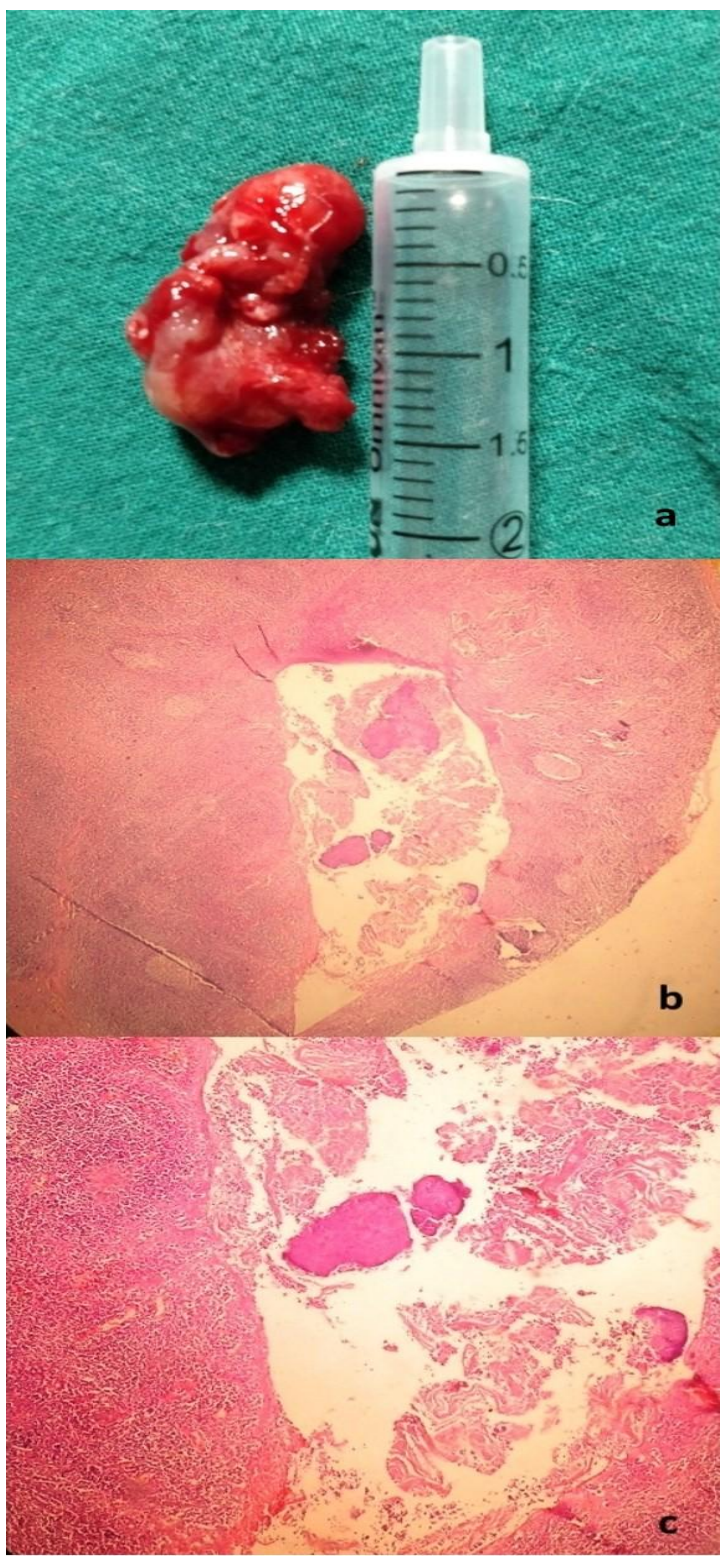

Fig.2 Post tonsillectomy a Gross specimen showing multiple swellings in the left tonsil. b \& c Histopathological examination under low and high power respectively showing tonsillar tissue within which are cavities lined by stratified squamous epithelium, containing lamellated keratin flakes, macrophages and lymphocytes

\section{Discussion}

Dermoid cysts have been classified by Meyer in 1955 as true dermoid, epidermoid and teratoid cysts. True dermoid cysts are lined with keratinized epithelium and contain skin appendages that could be described as hair follicles/sebaceous glands.EC are lined with simple squamous epithelium and its wall does not contain fibrous structures/skin appendages. 
Teratoid cysts may contain tissue ranging from simple squamous epithelium to ciliated cylindrical respiratory epithelium; its contents may be of ectodermal, mesodermal or endodermal origin. ${ }^{5}$ The term epidermoid cyst was coined by Roser in 1859. Few synonyms of EC are epidermal cyst/epithelial cyst/keratinous cyst/sebaceous cyst/milia/epidermal inclusion cyst/infundibular cyst. $^{2}$

EC can be either congenital or acquired, which are similar both clinically as well as histologically. The congenital ones are found in areas of embryonic fusion ie. epithelial remnants isolated during closure of the first and second branchial arches in the midline, whereas the acquired type occurs secondary to trauma/surgery. Various theories have been proposed regarding the origin of EC: theory of inclusion of ectodermal tissues during embryogenesis (Remark and Bucy,1854), metaplastic theory (Wendt,1873) which states that non keratinizing squamous epithelium lining the cavity undergoes metaplastic changes in response to prolonged irritation due to chronic infection, and lastly the implantation theory (Ewing,1928), which states that these cysts are a result of direct entry of epithelium into a site during trauma. ${ }^{6}$ Although an acquired epidermoid cyst would seem more likely in our patient in view of her age at presentation, she had no history of surgery/trauma.

The male:female ratio of patients with EC is 1:4.They can occur in any age group, starting from birth (congenital type) to 72 years; majority are aged between 10-35 years. ${ }^{4}$. This lead to the thought that cyst formation could be stimulated by hormonal influence during puberty. ${ }^{5}$ The fact that our patient was a 32 year-old female, also supports this thought.

EC appears as a painless, asymptomatic mass, slowly increasing in size or occasionally presents with throat complaints. ${ }^{1,2}$ However, our patient presented with the unusual complaint of earache, diagnosed as referred otalgia following normal otological examination. This is an uncommon presentation in these cases as evidenced by the review of literature. Referred otalgia ie. pain that is sensed by the ear but originating from a nonotologic source, poses a difficult diagnostic challenge to even the most experienced otolaryngologist. The ear receives an extensive sensory innervation via four cranial nerves $(\mathrm{V}, \mathrm{VII}, \mathrm{IX}, \mathrm{X})$ and two spinal segments $(\mathrm{C} 2, \mathrm{C} 3)$. The neuroanatomic basis of referred otalgia rests within one of these five general neural pathways. The most common causes are temporomandibular joint syndrome, pharyngitis, dental disease and cervical spine arthritis. If the patient presenting with otalgia has no local pathology, only by careful history and physical examination can all causes in this extensive differential diagnosis be excluded, as was done in our case, leading to the discovery of multiple swellings in the left palatine tonsil. Since the tonsil and tonsillar fossa are supplied by the glossopharyngeal nerve, any irritation or pain can be referred to the ear along the tympanic branch of the glossopharyngeal (Jacobson's) nerve. Acute pharyngitis, tonsillitis, peritonsillitis and peritonsillar abscess are common diseases that may cause referred otalgia. It also occurs frequently after tonsillectomy, but subsides when the pharynx heals. Lingual tonsillitis and foreign bodies (fish/chicken bones) embedded in the base of the tongue or tonsillar area, are other common causes of referred otalgia. ${ }^{3}$ Review of literature failed to elucidate tonsillar cyst as a cause for referred otalgia, but was seen in our case.

Diagnosis is confirmed by fine needle aspiration or excisional biopsy. ${ }^{2}$ The treatment for these lesions is surgical excision of the cyst. It should be excised without rupture because its contents could have an irritating effect on the surrounding fibrovascular tissue. Recurrence after surgery is rare. Malignant evolution has only been seen in the teratoid type and was reported to have an incidence of $0.5 \% .{ }^{4} \mathrm{~A}$ tonsillectomy was thus performed in our patient; the cyst was excised intoto and follow-ups were uneventful. Gross examination of the excised tonsil in our case 
showed multiple cysts which was unusual as evidenced in reviewed literature. ${ }^{1,2,4}$

Histopathologically, an EC is a cyst lined by stratified squamous epithelium with a granular layer filled with lamellated keratin material, which was seen in our case, confirming the diagnosis. ${ }^{2}$

\section{Conclusion}

Tonsillar involvement by epidermoid cysts is a rare occurrence. It can present with unusual complaints such as referred otalgia. All cases with otalgia should thus be examined thoroughly for the source of pain ie the pathology.

\section{References}

1. Shoba K, Kumar H, Raj D et.al. Epidermoid Cyst in Tonsil-A rare presentation. Transworld Medical Journal.2014;2(1):75-76.

2. Dutta M, Saha J, Biswas G et.al. Epidermoid Cysts in Head and Neck:Our Experiences, with Review of Literature. Indian J Otolaryngol Head \& Neck Surg.2013;65(1):14-21.

3. Kim KS. Referred Otalgia Induced by a Large Tonsillolith. Korean J Fam Med.2013;34(3):221-223.

4. Erol K, Erkan KM,Tolga D et.al. Epidermoid cyst localized in the palatine tonsil. Journal of Oral and Maxillofacial Pathology.2013;17(1):148.

doi:10.4103/0973-029X.110729.

5. Fernandez VJL, Rojas LJ, Fernandez AJ et.al. Dermoid cyst of the floor of the mouth. Acta Ororhinolaryngol Esp.2007;58 (1):31-33.

6. Pascal RJ, Mohan A, Abhilash AM. Epidermoid Cyst of Maxilla-Rare and interesting Case Report. Otolaryngology online journal.2013;3(4):163-170. 